\title{
YAP regulates PD-L1 expression in human NSCLC cells
}

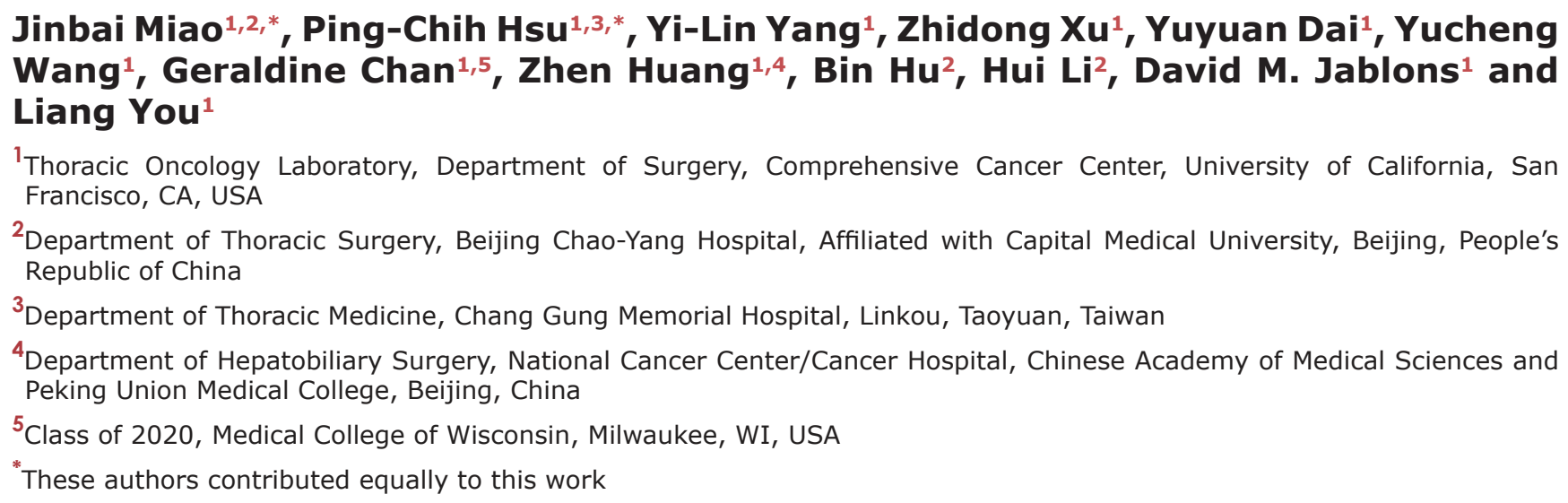

Correspondence to: Liang You, email: Liang.You@ucsf.edu

Keywords: programmed death-ligand 1; yes-associated protein; non-small cell lung cancer; hippo pathway

Received: July 26, $2017 \quad$ Accepted: November 13,2017_Published: December 09, 2017

Copyright: Miao et al. This is an open-access article distributed under the terms of the Creative Commons Attribution License 3.0 (CC BY 3.0), which permits unrestricted use, distribution, and reproduction in any medium, provided the original author and source are credited.

\section{ABSTRACT}

Programmed death-ligand 1 (PD-L1) is a membrane protein on tumor cells that binds to the PD-1 receptor expressed on immune cells, leading to the immune escape of tumor cells. Yes-associated protein (YAP) is a main effector of the Hippo/YAP signaling pathway, which plays important roles in cancer development. Here we show that YAP regulates PD-L1 expression in human non-small cell lung cancer (NSCLC) cells. First, we investigated YAP and PD-L1 expression at the protein level in 142 NSCLC samples and 15 normal lung samples. In tumor tissue, immunohistochemistry showed positive staining for YAP and PD-L1, which correlated significantly $(n=142$, $r=0.514, P<0.001)$. Second, in cell lines that express high levels of PD-L1 (H460, SKLU-1, and H1299), the ratio of p-YAP/YAP was lower and GTIIC reporter activity of the Hippo pathway was higher than those in three cell lines expressing low levels of PD-L1 (A549, H2030, and PC9) (P< 0.05). Third, in the same three cell lines, inhibition of YAP by two small interfering RNAs (siRNAs) decreased the mRNA and protein level of PD-L1 $(P<0.05)$. Fourth, forced overexpression of the YAP gene rescued the PD-L1 mRNA and protein level after siRNA knockdown targeting 3'UTR of the endogenous YAP gene. Finally, chromatin immunoprecipitation (ChIP) assays using a YAP-specific monoclonal antibody resulted in the precipitation of PD-L1 enhancer region encompassing two putative TEAD binding sites. Our results indicate that YAP regulates the transcription of PD-L1 in NSCLC.

\section{INTRODUCTION}

Lung cancer remains the leading cause of cancerrelated mortality worldwide [1,2], and non-small cell lung cancer (NSCLC) accounts for $80 \%$ of cases. Despite some improvements in treatment, the overall prognosis for NSCLC remains poor. The most common treatment plan for NSCLC is surgical resection combined with chemotherapy [3]. Platinum-based chemotherapy is the standard first-line treatment for advanced disease, but its role is limited due to its side effects. NSCLC patients acquire resistance to multiple therapeutic modalities, leading to a 5 -year survival rate of approximately $16 \%$ [4]. Targeted therapy has shown a better clinical effect for advanced NSCLC, but is currently only available for patients whose tumors have mutations such as epidermal 
growth factor receptor (EGFR) or anaplastic lymphoma kinase (ALK). The acquired resistance to targeted therapy also limits its ability to prolong survival [5]. Novel therapeutic strategies, including immunotherapy, are under investigation, but more useful biomarkers and therapeutic targets for patients with advanced NSCLC are needed.

PD-L1 (also known as B7-H1 or CD274), a type I transmembrane surface glycoprotein encoded by the CD274 gene, promotes T-cell tolerance and escapes host immunity by inhibiting CD8+ T-cell survival, effector function, and inducing Fas-mediated T-cell apoptosis [6]. PD-L1 is expressed on the surface of tumor cells, including in NSCLC, melanoma, and breast cancer. PD-L1 expression is significantly correlated with tumor related genes such as KRAS, p53, EGFR, and others $[7,8]$. In the tumor microenvironment, PD-L1 expression is greatly upregulated by LPS and interferon- $\gamma$ (IFN- $\gamma$ ) in a STAT1/3-dependent manner [9]. Although immune checkpoint inhibitors targeting the PD-1/PD-L1 pathway in NSCLC have shown promising results, with $\sim 30 \%$ of tumors responding [10-13], resistance is common. A better understanding of the regulatory mechanism of PD-L1 may help identify biomarkers and/or develop combinatorial strategies for clinical use.

Overexpression of the transcription coactivator Yesassociated protein (YAP) has been found in many cancers due to abnormal amplification, loss of Hippo signaling by mutation, and/or downregulation of the core Hippo component [14]. YAP overexpression also contributes to self-renewal, tumor initiation capacity and resistance to anticancer drugs [15-18]. The role of YAP in tumor immunity has just begun to be explored [19]. The study by Moroishi et al. [14] indicates that hyperactivation of YAP and TAZ significantly contributes to tumor growth suppression through TEAD-mediated transcription. Another study found that YAP is a negative regulator of innate immunity through interaction with IRF3 [20]. Although these limited studies have demonstrated that Yap plays a role in tumor immunity, the effects of YAP on tumor growth, especially in the context of reciprocal interactions between tumor cells and host anti-tumor immune responses, remain largely unknown because of the complexity of tumorigenesis and immune regulation.

In this study, we hypothesized that YAP is involved in the regulation of PD-L1 expression in NSCLC. To test this, we evaluated YAP and PD-L1 expression in human NSCLC tissues and then investigated whether PD-L1 is a downstream target of YAP in NSCLC cell lines.

\section{RESULTS}

\section{YAP and PD-L1 are co-expressed in NSCLC tissues}

YAP is an important mediator protein in cancer development. Programmed death-ligand 1 (PD-L1; also called B7-H1 or CD274), which is expressed on many cancer cells, is one of the most important inhibitory molecules that promote tumor immune escape. To investigate the relationship of YAP and PD-L1 in NSCLC tissues, we used immunohistochemistry (IHC) to examine their protein level in 142 samples of primary human NSCLC and 15 normal lung samples (Figure 1). We found that 88 cases $(62.0 \%)$ were YAP-positive and 52 cases (36.6\%) were PD-L1 positive (Table 1, Supplementary Table 1). Forty-three cases (30.3\%) were both YAP and PD-L1 positive, accounting for, respectively, $48.9 \%$ of YAP-positive samples and $82.7 \%$ of PD-L1 positive samples. PD-L1 positive samples showed significantly higher YAP positive ratios compared to $\mathrm{PD}-\mathrm{L} 1$ negative samples $\left(\mathrm{X}^{\mathrm{s}}=14.947, P<0.001\right)$. In normal lung tissues, no cases were positive for YAP or PD-L1 (Table 2). There was no significant difference in YAP and PD-L between different pathological types and TNM stage $(P>0.05)$ (Supplementary Tables 2, 3). To further analyze the relationship between YAP and PD-L1, we performed a Spearman product correlation test. YAP and PD-L1 were mildly, but still significantly correlated at the protein level $(n=142, \mathrm{r}=0.514, P<0.001)$.

\section{YAP and PD-L1 are co-expressed in NSCLC cell lines}

To elucidate the relationship between YAP and PD-L1, we further studied eight NSCLC cell lines: H460, H2170, SKLU-1, H1975, H1299, A549, H2030 and PC9. We analyzed GTIIC reporter activity of Hippo pathway and found higher activity in H460, H2170, SKLU-1, H1299, H1975, and H2030 cell lines than in A549, PC9 and LP-9 $(P<0.05$; Figure 2C, Supplementary Table 6$)$. We then used qRT-PCR to detect mRNA expression. In SKLU1 and H1299 cell lines, the PD-L1 and YAP mRNA levels were significantly higher than in the other cell lines $(P<0.05$; Figure 2B, Supplementary Table 5$)$. In H460 cells, PD-L1 mRNA expression was the highest $(P<0.001)$, but YAP mRNA expression was lower than that in SKLU-1 and H1299 cell lines $(P<0.001)$, higher than that in A549, H2170 and $\mathrm{H} 2030$ cell lines $(P<0.01)$, and the same as that in H1975 and PC9 cell lines (Figure 2A, Supplementary Table 4). Next, we used western blot to detect protein expression, and found that the p-YAP (ser127) /YAP ratio decreased significantly in cell lines that expressed high levels of PDL1 (H460, SKLU-1, and H1299) (Figure 2D and 2E). YAP was stained in both nucleus and cytoplasm, whereas pYAP (ser127) was found in the cytoplasm (Supplementary Figure $1 \mathrm{~A}, 1 \mathrm{~B}, 1 \mathrm{E})$. Then we detected protein expression of pYAP (Tyr357), src, and TAZ in H2030, PC9 and A549 cells with different degrees of YAP and pYAP (ser127) expression. The protein expression level of p-YAP (ser127) was higher than that of pYAP (Tyr357) in H2030 and PC9 cell lines. In A549 cell lines, the expression of pYAP (Tyr357) was higher than 
in H2030 and PC9 cell lines. (Supplementary Figure 1C). These results suggest that YAP and PD-L1 are co-expressed in H460, SKLU-1, and H1299 cell lines.

\section{Inhibition of YAP downregulates PD-L1 expression in H460, SKLU-1, and H1299 cell lines}

To further understand whether YAP can regulate PDL1, siRNA-YAP (3' and 5') was used to silence the YAP gene in H460, SKLU-1 and H1299 cell lines. The mRNA and protein levels of PD-L1 were detected, respectively, by qRT-PCR and Western blot. The results confirmed that the YAP gene was significantly inhibited by transfection of siRNA-YAP (Figure 3A). qRT-PCR showed a significant decrease in PD-L1 mRNA levels in the H460, SKLU-1 and H1299 cell lines after 48 hours of treatment with siRNA-YAP ( $3^{\prime}$ and 5'UTR) $(P<0.05)$ (Figure 3B). Western blot confirmed that the protein expression was also reduced (Figure 3C). After YAP gene silencing,
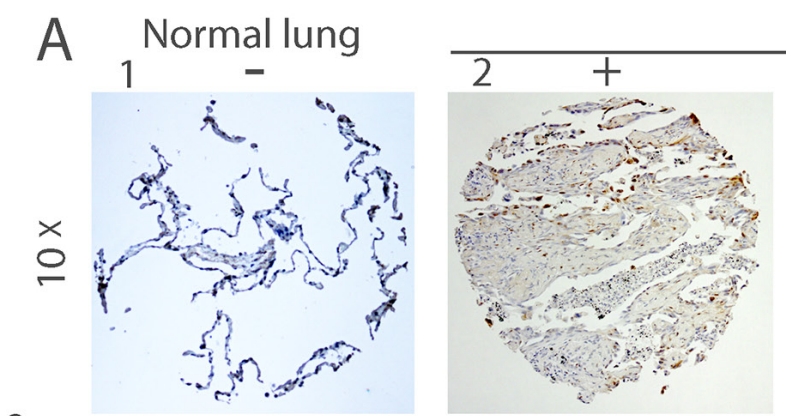

NSCLC tissues

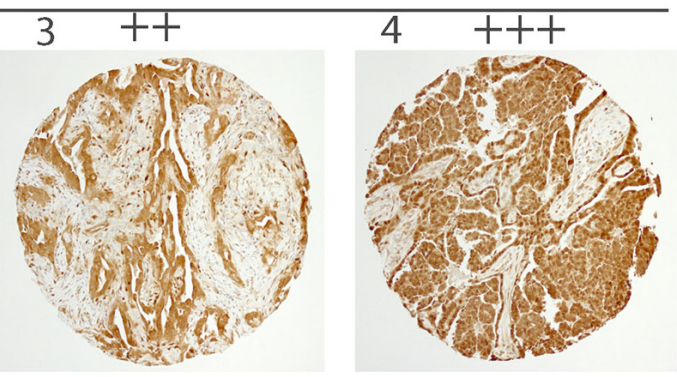

$\frac{2}{2}$

NSCLC tissues
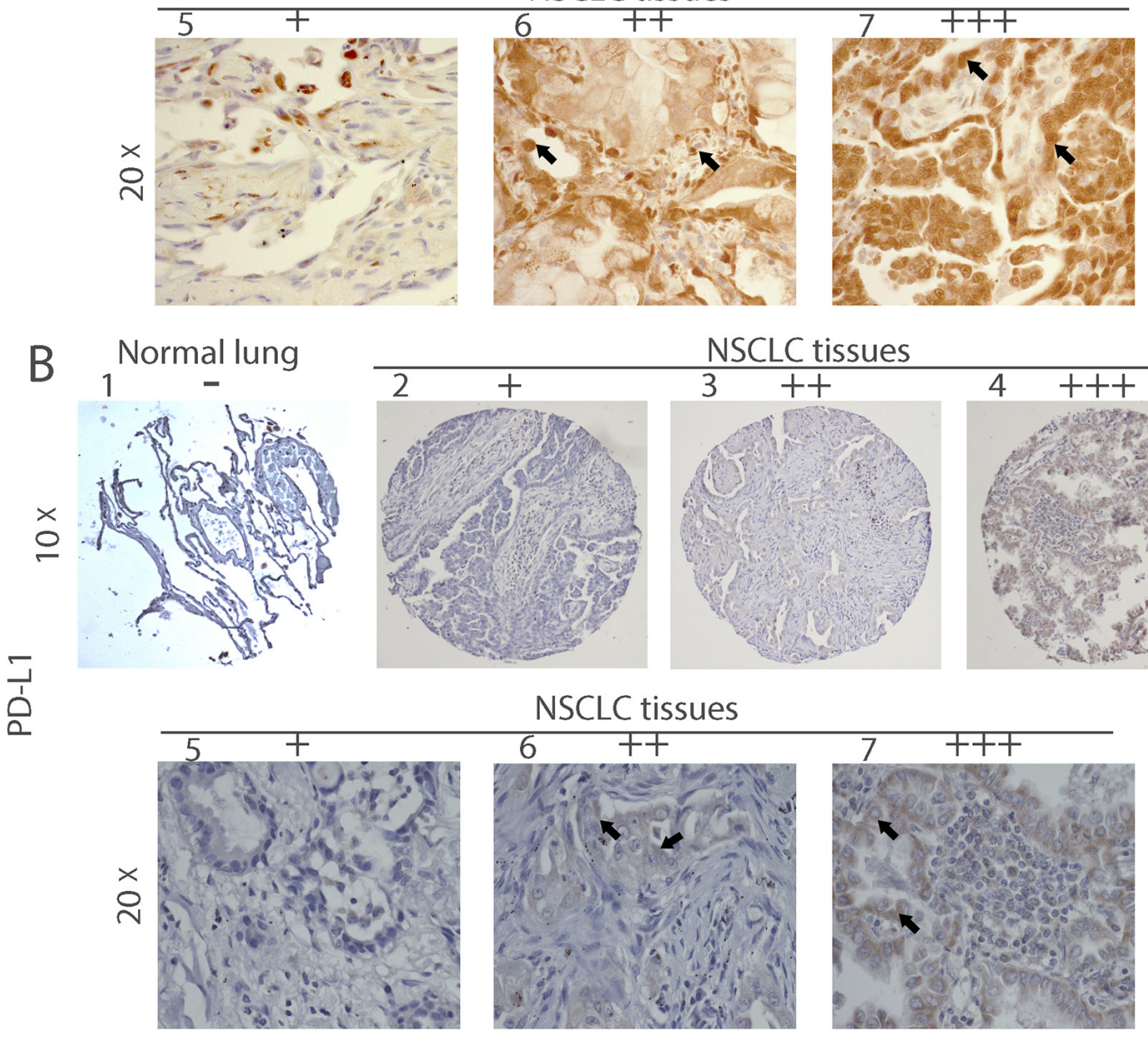

Figure 1: Immunohistochemistry of YAP and PD-L1 in human NSCLC tissues. Representative image showing expression of YAP protein (A) and PD-L1 protein (B) in human NSCLC tissues and normal lung tissues analyzed by immunohistochemistry. (A:1) and (B:1) are normal lung tissues. (A:2-7) and (B: 2-7) are NSCLC tissues. (A:5-7) Staining of YAP was localized in nuclei (arrow) and (B:5-7) staining of PD-L1 was localized in membrane (arrow), under a $20 \times$ objective lens. - and + mean negative; ++ and +++ mean positive. 
Table 1: YAP and PD-L1 IHC comparison in 142 human primary NSCLC tissues

\begin{tabular}{lcccccc}
\hline & \multicolumn{5}{c}{ YAP } & \\
\hline & & Positive $(++/+++)$ & Negative $(-/+)$ & Total & $\mathbf{X}^{2}$ & $\boldsymbol{P}_{\text {value }}$ \\
\hline \multirow{2}{*}{ PD-L1 } & Positive $(++/+++)$ & 43 & 9 & 52 & \\
& Negative $-/+)$ & 45 & 45 & 90 & 14.947 & $<0.001$ \\
\hline Total & & 88 & 54 & 142 & 14 \\
\hline
\end{tabular}

$-=$ no stain $;+=$ weak stain $;++=$ moderate stain $;+++=$ strong stain $;++/+++=$ positive; $-/+=$ negative .

Table 2: YAP and PD-L1 IHC comparison in normal human lung tissues

\begin{tabular}{lcccc}
\hline & & YAP & \\
\hline & & Positive $(++/+++)$ & Negative (-/+) & Total \\
PD-L1 & Positive $(++/+++)$ & 0 & 0 & 15 \\
\hline Total & Negative $(-/+)$ & 0 & 0 & 15 \\
\hline
\end{tabular}

$-=$ no stain $;+=$ weak stain $;++=$ moderate stain $;+++=$ strong stain $;++/+++=$ positive $;-/+=$ negative .

the protein expression of YAP and pYAP (ser127) were significantly decreased, but the protein expression levels of Src, TAZ, and pYAP (Tyr357) were no significantly changed (Supplementary Figure 1D). Together, these results suggest that $\mathrm{PD}-\mathrm{L} 1$ expression is regulated by YAP expression in NSCLC cells.

\section{Forced overexpression of the YAP gene rescued PD-L1 mRNA and protein levels}

To confirm that YAP regulates PD-L1 transcription and translation, we analyzed PD-L1 mRNA and protein levels after YAP inhibition and/or forced overexpression of the YAP gene in H460 cells. We used YAP siRNA, which targeted the 3'UTR end of the YAP gene. After YAP depletion, the PDL1 mRNA and protein levels were decreased, similar to what occurred after we inhibited YAP by using pooled YAP siRNA. To verify that the decreased PD-L1 expression was regulated by YAP knockdown, we examined PD-L1 expression level after forced YAP overexpression in siRNA-YAP H460 cell line. Using qRT-PCR and western blot analyses, we confirmed the increase in YAP mRNA and protein levels after forced YAP overexpression $(P<0.01)$ (Figure 4). These findings confirm that the YAP gene regulates PD-L1 mRNA and protein expression.

\section{YAP regulates $\mathrm{PD}-\mathrm{L} 1$ at the transcriptional level through binding to the PD-L1 enhancer}

YAP as a transcription co-activator, together with TEAD family proteins, regulates many genes, including BIRC5, CTGF and CYR61 [21]. Therefore, we asked whether YAP regulates PD-L1 at the transcriptional level. To answer this question, we examined the PD-L1 enhancer region (-10000 bps) upstream of the transcription starting site of PD-L1 and found two putative TEAD-binding sites (CATTCC), which are 7941 bps and 7911 bps upstream of the PD-L1 transcription start site (Figure 5A). We used chromatin immunoprecipitation (ChIP) to test our hypothesis in H460, SKLU-1 and H1299 cells. ChIP studies using a YAP specific monoclonal antibody resulted in the precipitation of two PD-L1 enhancer regions encompassing the putative TEAD binding site (Figure 5B). In the control ChIP assays using Rabbit IgG or without any antibody, we did not detect PD-L1 enhancer-region binding. Then we investigated the transcriptional activity change after YAP binding to PD-L1 in A549, H1975, H1299 and SKLU-1 cell lines, which express different levels of PD-L1. The results indicate that transcriptional activity differed significantly among cell lines, which was consistent with the results of Western blot expression (Figure 5C). These findings confirmed that YAP directly occupied the enhancer regions of PD-L1.

\section{DISCUSSION}

The results of our study provide several lines of evidence to support that YAP is involved in the regulation of PD-L1 expression. First, IHC staining indicated that YAP and PD-L1 expression were significantly correlated in NSCLC tissue. Second, GTIIC reporter activity of the Hippo pathway was higher and the ratio of P-YAP/YAP was lower in cell lines that expressed high levels of PDL1 (H460, SKLU-1, and H1299) than in other cell lines. Moreover, in H460, SKLU-1 and H1299 cells, inhibition of the endogenous YAP by siRNAs downregulated PDL1 mRNA and protein levels, and this could be rescued by forced overexpression of YAP. Third, our ChIP results demonstrated that precipitation of the PD-L1 enhancer region encompasses the putative TEAD binding site in H460, SKLU-1 and H1299 cell lines.

In our study, YAP and PD-L1 were expressed to different degrees in NSCLC tissues. YAP, a candidate oncogene in a variety of cancers, is significantly elevated in hepatocellular cancer, prostate cancer, and breast carcinoma $[22,23]$. The positive rate of YAP was $62.0 \%$ 
in our study, which is similar to reported rates of $54 \%$ [23] and 66.3\% [24]. The PD-L1 ligand of programmed cell death 1 (PD-1) is upregulated in various types of cancers [25]. In recent clinical series, PD-L1 expression

A
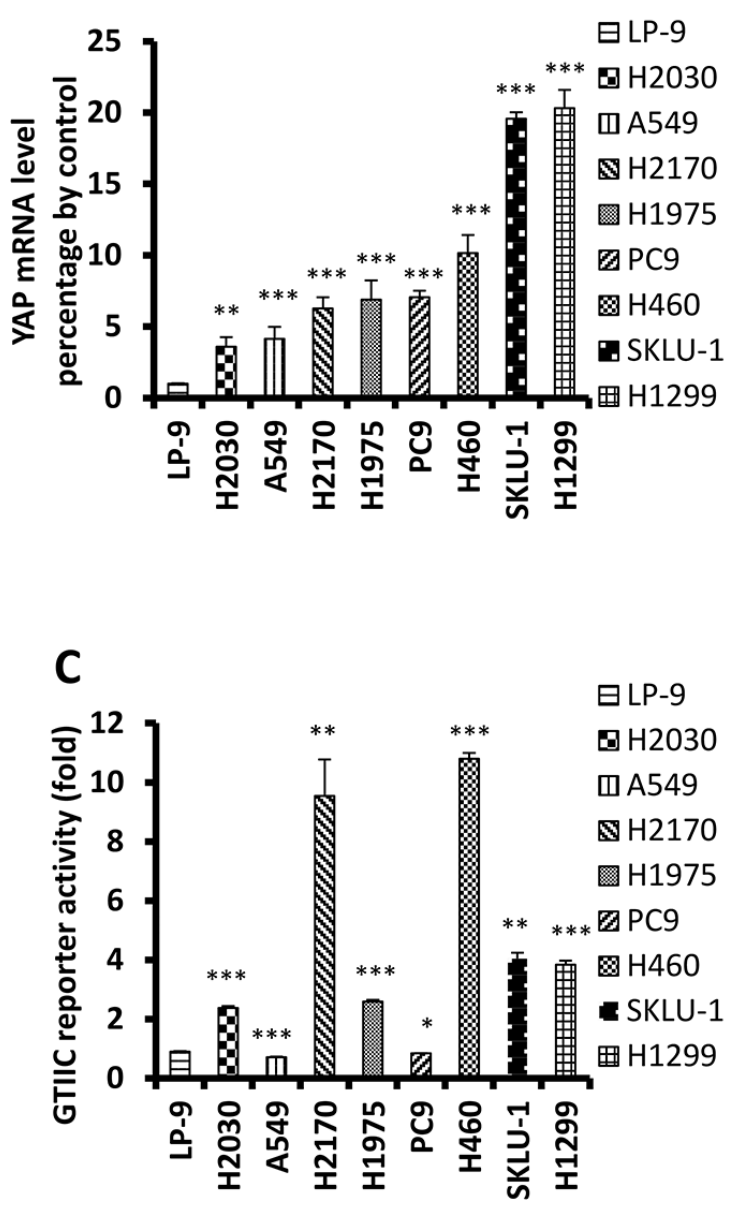

in NSCLC ranges from $7.4 \%$ to $72.7 \%[22,26]$, possibly because of clinicopathologic characteristics and/or molecular alterations. In an analysis of 297 NSCLC cases, $43.1 \%$ of NSCLC patients had PD-L1 positive

B
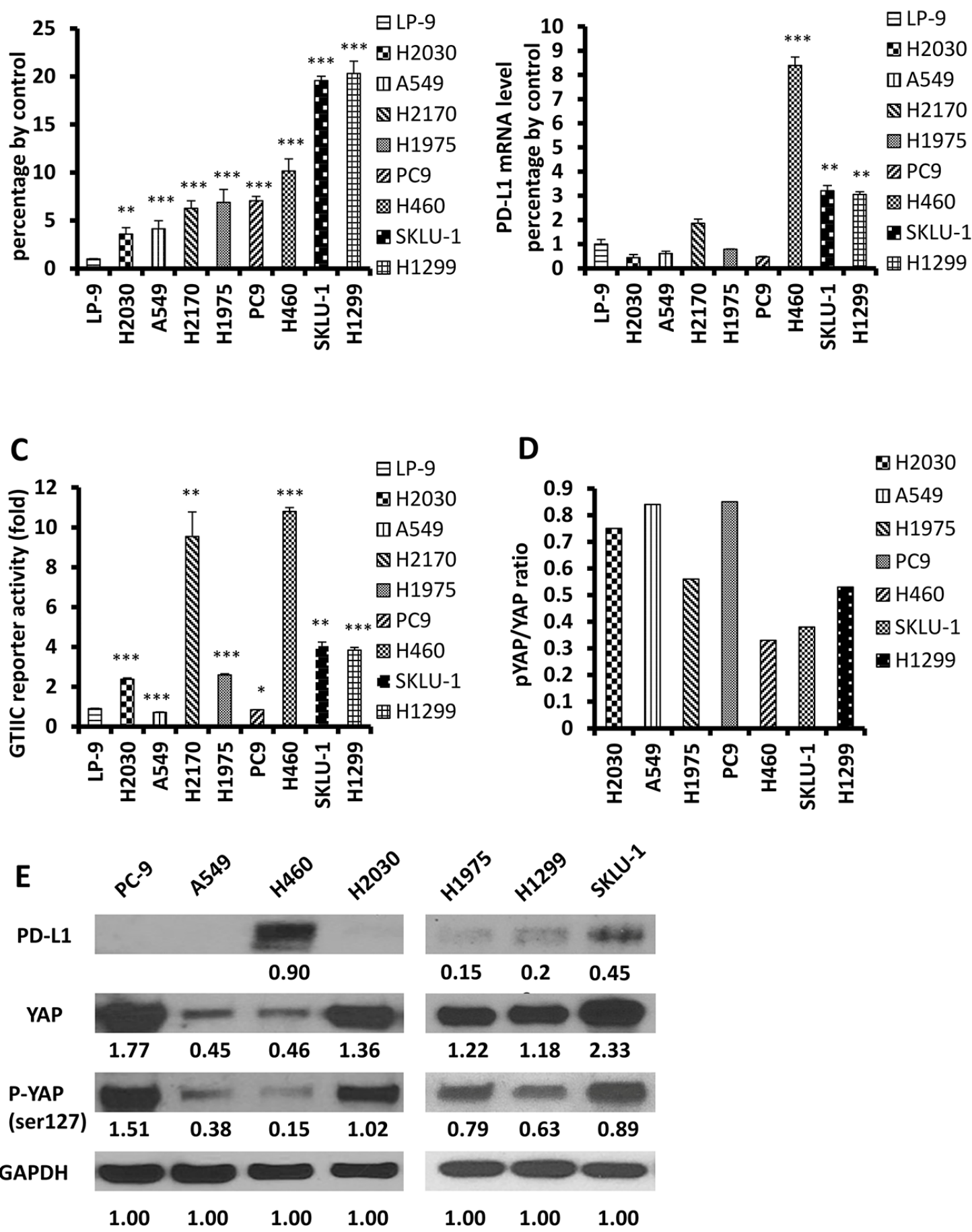

Figure 2: Expression of YAP and PD-L1 in NSCLC cell lines. (A-B) The mRNA levels of YAP and PD-L1 in NSCLC cell lines were measured using qRT-PCR, and LP-9 cell line was used as control $(\mathrm{F}=174.10 P<0.001 ; \mathrm{F}=635.77 P<0.001)$. VS LP-9: ${ }^{*} P<0.05$, ${ }^{* *} P \leq 0.01,{ }^{* * *} P \leq 0.001$. (C): GTIIC reporter activity of the Hippo pathway in NSCLC cell lines, and LP-9 cell line was used as control (F $=311.39 ; P<0.001)$. VS LP-9: ${ }^{*} P<0.05,{ }^{* *} P \leq 0.01,{ }^{* * *} P \leq 0.001$. (D) pYAP/YAP ratio in NSCLC cell lines based on the value of Western blot. (E) Western blot was used to detect levels of YAP, pYAP and PD-L1 in NSCLC cell lines. GAPDH was detected as a loading control. Band intensity was analyzed with ImageJ software and normalized with the intensity of GAPDH band. 
staining [27]. In our study, the positive rate of PD-L1 was $36.6 \%$ (52/142), and TNM stage and histopathology did not significantly differ between PD-L1 positive and PDL1 negative patients $(P>0.05)$. In tumor development, YAP is thought to be associated with a variety of factors. A significant correlation between YAP and Jag-1 staining was observed in pancreatic ductal adenocarcinoma $(r=0.442, p<0.001)$ [28]. However, YAP and PD-L1 coexpression in NSCLC has not been reported previously. Our study showed this co-expression in 30.3\% (43/142) of NSCLC samples and that the YAP-positive ratio was higher in PD-L1 positive samples than in PD-L1 negative samples $(P<0.001)$. Expression of the two proteins was mildly, but still significantly correlated $(n=142, r=0.514$, $P<0.001)$.

We investigated eight NSCLC cell lines and found that three (H460, SKLU-1 and H1299) had higher PDL1 expression in mRNA and protein level compared to others. Chen et al. [29] reported different expression levels of PD-L1 in PC9 and H1975 cell lines. In our study, the degree of PD-L1 expression was lower in H1975 than in H460, SKLU, and H1299, which was similiar to Chen et al's western blot results. In their study, PD-L1 expression in H1975 cells was also stronger than in PC9 cells. We also found that high expression of PD-L1 is positively correlated with an increase of intranuclear YAP. YAP activation plays an important role in transcriptional regulation. Phosphorylation of YAP by LATS1 leads to cytoplasmic retention of YAP, which inhibits its function as a nuclear transcription coactivator [30-32]. In cell lines that express high levels of PD-L1, we found that the ratio of p-YAP/YAP was lower and GTIIC reporter activity of the Hippo pathway was higher than in cell lines that express low levels of PD-L1. The expression of pYAP (Tyr357) was weak in the cell lines we studied, which suggested that its effect may be limited in the cell lines
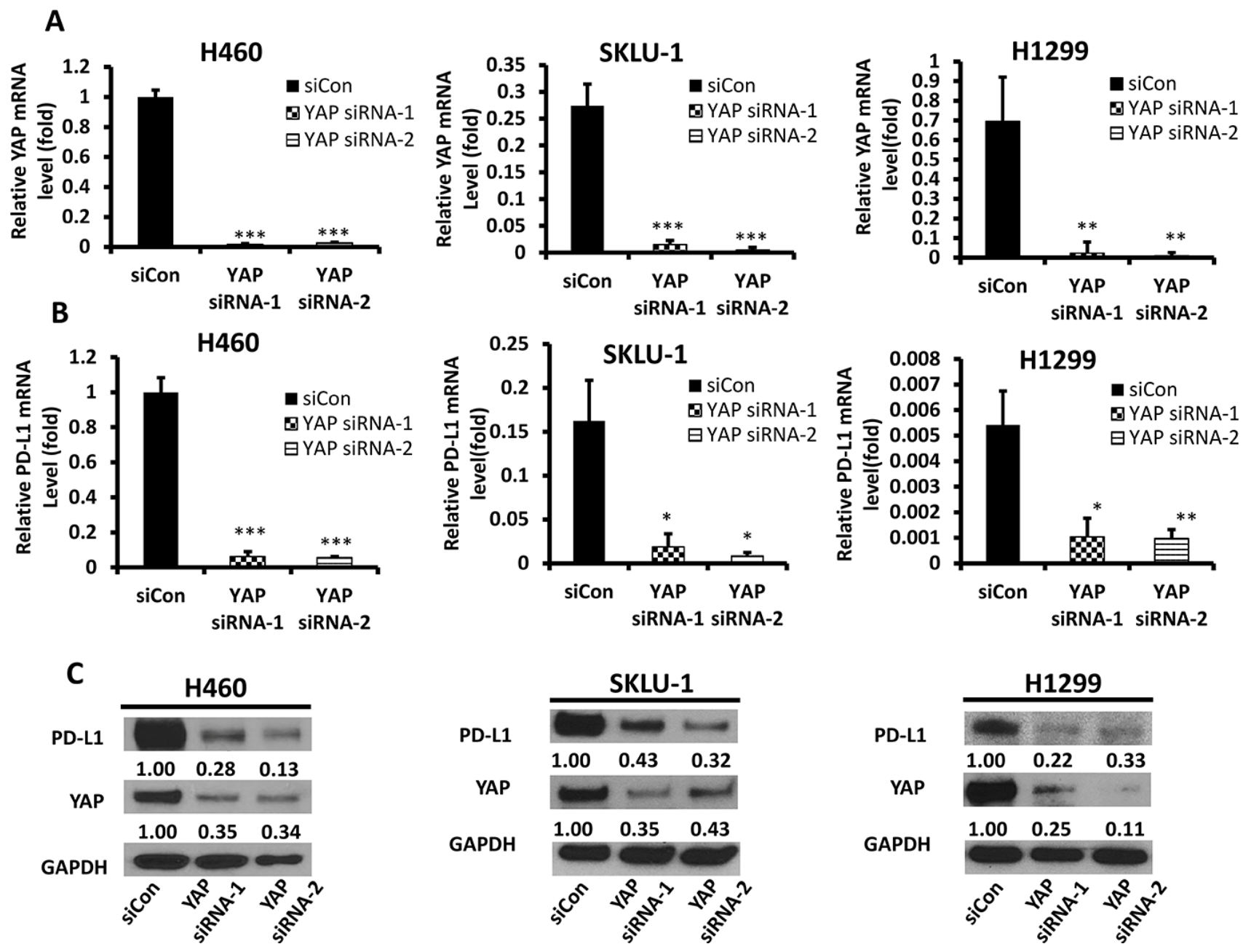

Figure 3: YAP and PD-L1 expression in H460, SKLU-1 and H1299 cell lines after knockdown of YAP. (A) YAP mRNA level in H460, SKLU-1 and H1299 cells after YAP inhibition by siRNA1,2 was measured using qRT-PCR. (B) PD-L1 mRNA level in H460, SKLU-1 and H1299 cells after YAP inhibition by siRNA1,2 was measured using qRT-PCR. (C) Western Blot analysis in H460, SKLU-1 and H1299 cell lines transfected with YAP siRNA1,2 or control siRNA. GAPDH was detected as a loading control. Band intensity was analyzed with ImageJ software and normalized with the intensity of GAPDH band. YAP-knockdown reduced PD-L1 expression significantly. ${ }^{*} P<0.05,{ }^{* *} P \leq 0.01,{ }^{* * *} P \leq 0.001$. 


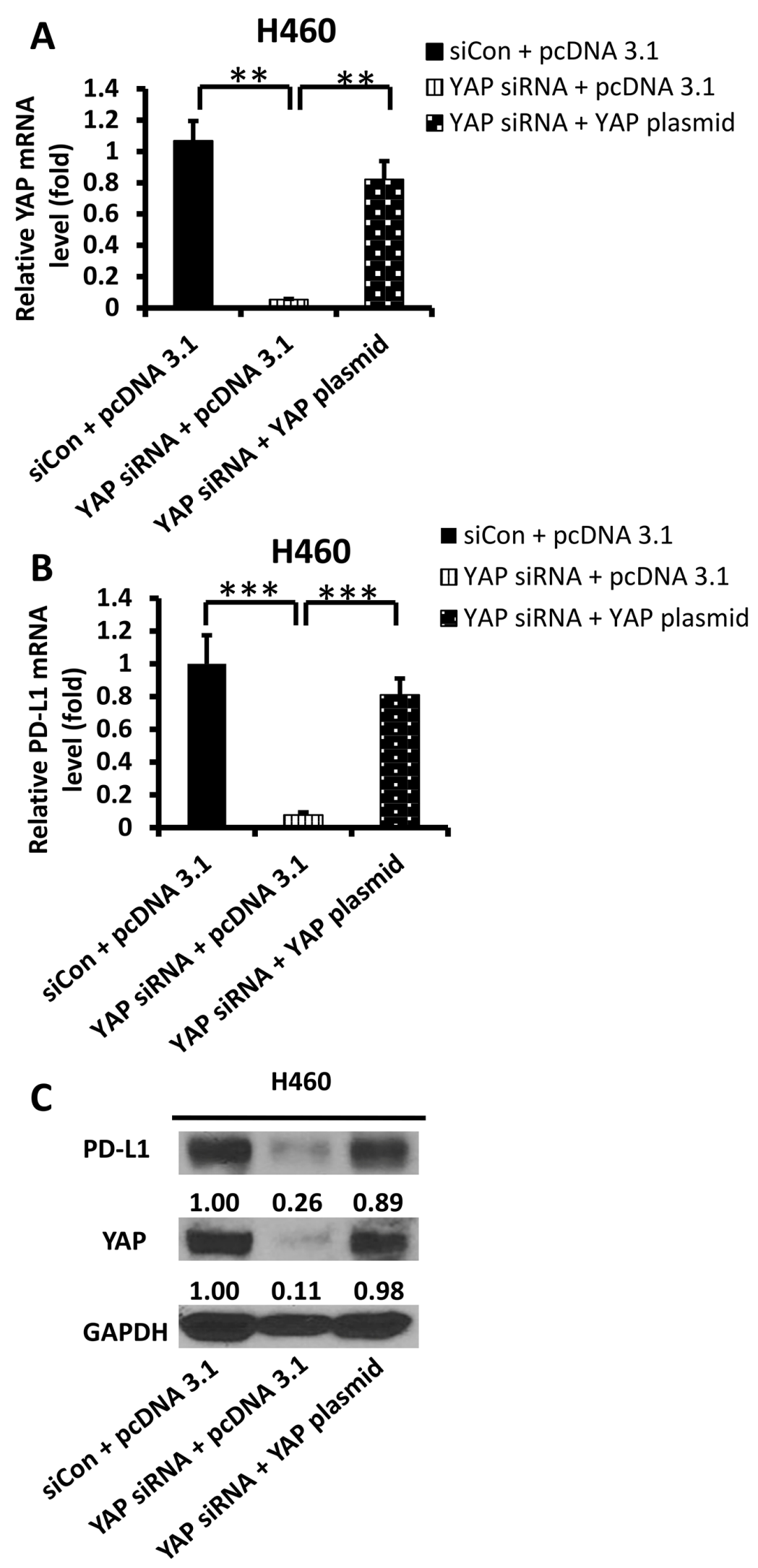

Figure 4: Expression of PD-L1 and YAP after YAP forced over-expression in siRNA-YAP H460 cells. (A-B) qRT-PCR analysis of mRNA levels of YAP and PD-L1 after YAP silencing by siRNA and/or forced over-expression of the YAP gene in H460 cells. (C) Western blot analysis of YAP, PD-L1 after YAP silencing by siRNA and/or forced over-expression of the YAP gene in H460 cells. GAPDH was detected as a loading control. Band intensity was analyzed with ImageJ software and normalized with the intensity of GAPDH band. ${ }^{*} P<0.05,{ }^{* *} P \leq 0.01,{ }^{* * *} P \leq 0.001$. 
we studied. Furthermore, silencing the YAP gene with siRNA-YAP ( $3^{\prime}$ and $5^{\prime}$ ) significantly decreased PD-L1 transcriptional level. PD-L1 expression levels were also significantly rescued after forced YAP overexpression in siRNA-YAP H460 cells. These findings suggest that YAP is involved in the positive regulation of $\mathrm{PD}-\mathrm{L} 1$ expression in NSCLC cells.

YAP translocation into the nucleus, as a transcriptional coactivator, plays an oncogenic function mainly by interacting with the TEAD family of transcription factors [33, 34]. Accumulating evidence [35-38] suggests YAP expression promotes cell transformation, epithelial-to-mesenchymal transition (EMT), and cell invasion. Our previous studies have shown that YAP promotes drug resistance in NSCLC through an autocrine loop with the ERBB3 pathway, regulation of $\mathrm{ABCG} 2$, and side-population cell formation $[15,39]$. However, the role of YAP in tumor immunity is poorly understood. A recent study [40] reported that YAP regulates the transcription of PD-L1 in EGFRTKI-resistant PC9 cells through binding of the YAP/ TEAD complex to the PD-L1 promoter, but it is unclear whether this mechanism exists in other NSCLC cell lines. The heterogeneity of PD-L1 expression levels and the promising approaches for targeted immunotherapy lung cancer treatment make it important to understand the signaling regulation of PD-L1 expression in NSCLC cells [41]. Here our results indicate that at the transcription level, YAP regulates PD-L1 expression in NSCLC cell lines. Our ChIP data performed with H460, SKLU1 and H1299 cells demonstrate that YAP binds to the PD-L1 enhancer and regulates the expression of PD-L1 transcription, and the level of PD-L1 expression was consistent with YAP transcriptional activity in A549, H1975, H1299 and SKLU-1 cell lines.

Expression of PD-L1 as an adaptive response to endogenous antitumor immunity can occur because PD-L1 is induced on most tumor cells in response to inflammatory cytokines, predominantly IFN- $\gamma$, which has been confirmed in melanoma, ovarian cancer, and part of lung cancer [42]. IFN- $\gamma$ is a pro-inflammatory cytokine that regulates anti-tumor immunity and is produced mainly by activated type 1 CD4+ helper T cells (Th1), CD8+ cytotoxic T lymphocytes (CTLs), macrophages, and natural killer (NK) cells. The IFN- $\gamma$-induced PD-L signaling pathway may be involved with interferon regulatory factor-1, STAT 1/3 [43, 44]. The regulation of PD-L1 appears to be very complex and may involve multiple signaling pathways. Feng et al. [45] showed that lactic acid can promote PD-L1 expression and that TAZ

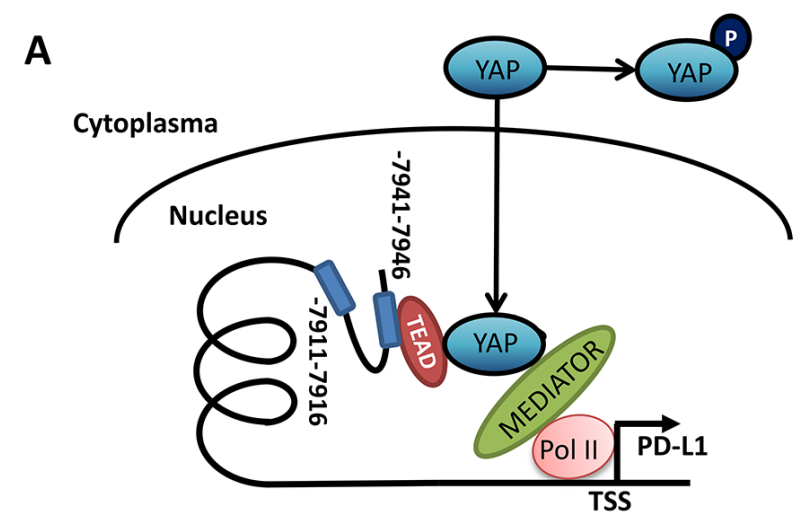

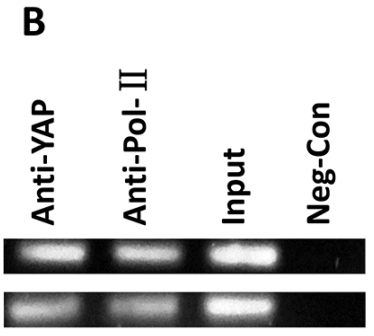

SKLU-1

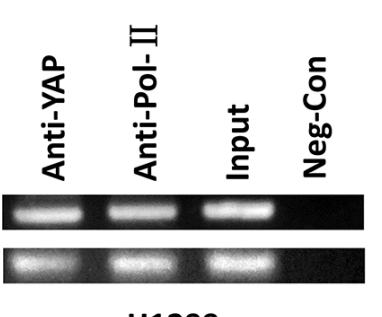

H1299
C

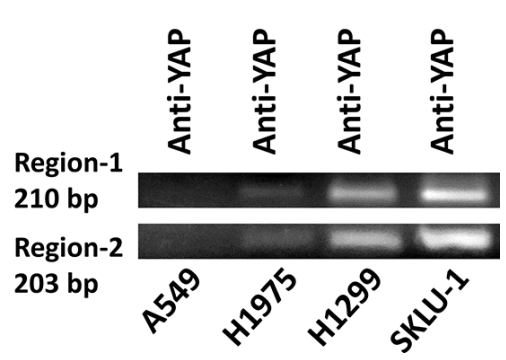

Figure 5: YAP regulates PD-L1 at the transcriptional level through binding to PD-L1 enhancer. (A) Schematic of the PDL1 promoter region. Sequence analysis revealed a putative YAP1-TEAD co-binding site between -7911 to -7941 nucleotides upstream of the transcription start site. (B) ChIP assays were performed with SKLU-1 and H1299 cells in two regions (203 bp and 210 bp), which were shown by gel bands of RT-PCR products with 36 cycles. (C) ChIP assays were performed with A549, H1975, H1299 and SKLU-1 cells in two regions (203 bp and $210 \mathrm{bp}$ ), which were shown by gel bands of RT-PCR products with 30 cycles. 
contributes to $\mathrm{PD}-\mathrm{L} 1$ regulation. We found that after YAP gene silencing, PD-L1 expression decreased significantly, but TAZ expression did not change. Immune checkpoints including PD-L1/PD-1 have are of great interest for researchers of anticancer immunotherapy. Our finding that YAP regulates PD-L1 expression in NSCLC cell lines provides a basis for exploring potential therapeutic Hippo/ YAP targets. PD-L1 inhibitors combined with drugs that inhibit YAP1 activity may have a synergistic effect. Drugs that potentially regulate YAP activity include Dasatinib, Y27632, TPCA-1, AZD0532, and so on, which target YES1, GPCR-ROCK, stat3, Src/YES, and other sites [46]. Recently, the combination of the inhibitor of YAP1 activity with other drugs have shown some promising results in EGFR-mutant NSCLC mouse models [47]. Further study of the interactions between Hippo/YAP signaling pathways and immunomodulation could lead to the development of new synergistic drugs for lung cancer.

In conclusion, our study indicates that YAP and PD-L1 expression are significantly correlated in NSCLC tissues. The reason for this may be that YAP regulates the expression of PD-L1 in NSCLC at the transcriptional level. Future studies should focus on whether regulation of YAP and PD-L1 is active in a variety of tumor types and whether this should affect the choice of clinical treatment and prognosis for NSCLC in the future.

\section{METHODS}

\section{Cell culture}

Human NSCLC cell lines H460, H2170, SKLU-1, H1975, H1299, A549, H2030, and PC-9 were obtained from American Type Culture Collections (Manassas, VA). Cell lines were maintained in RPMI-1640 except for SKLU-1, which was maintained in EMEM. All media were supplemented with $10 \%$ heat-inactivated fetal bovine serum, penicillin $(100 \mathrm{mg} / \mathrm{ml})$. LP-9 was maintained in M199 supplemented with $15 \%(\mathrm{v} / \mathrm{v})$ heat-inactivated FBS, $10 \mathrm{ng} / \mathrm{ml} \mathrm{EGF}, 0.4 \mathrm{ug} / \mathrm{ml}$ hydrocortisone and penicillin (100 IU/ml). All cells were cultured at $37^{\circ} \mathrm{C}$ in a humid incubator with $5 \% \mathrm{CO}_{2}$.

\section{RNA isolation, cDNA synthesis and quantitative real-time RT-PCR}

Total RNA was extracted from cells using the RNeasy Mini kit (Qiagen, Valencia, CA). The cDNA was transcribed from $500 \mathrm{ng}$ of total RNA using iScriptcDNA Synthesis Kits (Bio-Rad, Hercules, CA), according to the manufacturer's protocol. The cDNA was used as the template for real-time PCR detection using TaqMan Technology on an Applied Biosystems 7000 sequence detection system (Applied Biosystems, Foster City, CA). Expression of PD-L1, YAP genes and endogenous control gene b-glucuronidase (GUSB) was detected by using the primer and probe sequences commercially available (Thermo Fisher Scientific, Rockford, IL) and analyzed using Relative Quantification Software (Applied Biosystems). The TaqMan Assay IDs of primer sequences for qRT-PCR were GUSB (Hs00939627 m1, Lot 1516740 , 1516749 G3), PD-L1 (CD274, Hs01125301 m1, Lot 1548518, 1548524 D12) and YAP1 (Hs00902712 g1; Lot 1450809; 1450830 H6). Waltham, MA USA 02451.

\section{SiRNA and plasmid DNA transfection}

The SMAR-Tpool siRNA targeting YAP (YAP siRNA-1) was purchased from Thermo Scientific Dharmacon (Pittsburgh, PA, USA). Non-targeting siRNA was used as control (Thermo Scientific Dharmacon). Another YAP siRNA targeting the 3'UTR end of the YAP gene (YAP siRNA-2) was purchased from Life Technologies (Grand Island, NY). The YAP plasmid DNA used to overexpress the YAP gene in the cells was purchased from Addgene (Cambridge, MA). Cells were plated in 6-well plates (for western blot) and 24-well plates (for PCR and reporter assay) for 24 hours before treatment. Cells were transfected with $4 \mu \mathrm{g}$ of YAP plasmid DNA using Lipofectamine 2000 (Invitrogen, Carlsbad, CA) transfection reagent, and $100 \mathrm{nmol} / \mathrm{L}$ of siRNA using Lipofectamine RNAiMAX (Invitrogen) according to the manufacturer's protocol. After transfection for 48 hours, cells were harvested for further analysis.

\section{Luciferase reporter assay}

The $8 \times$ GTIIC-luciferase plasmid (Addgene) and Renilla luciferase pRL-TK plasmid (Promega, Madison, WI) were co-transfected into cell lines. The si-RNA transfection reagent was Lipofectamine RNAiMAX (Invitrogen). After 48 hours, cells were harvested and transferred into a 96-well plate for analysis by using the Dual-Luciferase Reporter Assay Kit (Promega). Luminescent signaling was detected on a GloMax-96 Microplate Luminometer (Promega) according to the manufacturer's instructions.

\section{Western blot analysis}

The following primary antibodies were used for immunoblot analysis: YAP, phospho-YAP (Ser127), Src, Lamine-b and PD-L1 from Cell Signaling, Inc. (Danvers, MA); phospho-YAP (Tyr357) from Abcam (Cambridge, MA) and Sigma-Aldrich (St. Louis, MO); TAZ and $\alpha$-tubulin from Santa Cruz Biotechnology (Santa Cruz, CA). Total protein was extracted from cell lines using M-PER Mammalian Protein Extraction Reagent (Thermo Fisher Scientific, Rockford, IL), and nuclear/cytoplasm proteins extracted using a nuclear/cytoplasm extraction kit (Thermo Fisher Scientific Inc.) were supplied with Complete Protease Inhibitor Cocktails (Roche, Lewes, UK), according to the manufacturers' protocols. The protein concentrations were 
measured with the Pierce BCA Protein Assay Kit (Thermo Fisher Scientific, Rockford, IL). A total of $15 \mu \mathrm{g}$ of proteins was run on $4 \sim 20 \%$ gradient SDS-polyacrylamide gels (Bio-Rad) and transferred to Immobilon-P nitrocellulose membranes (Millipore, Bellerica, MA). The membranes were blocked in 5\% non-fat milk and then probed with the primary antibodies overnight at $4{ }^{\circ} \mathrm{C}$. The membranes were incubated with appropriate secondary antibodies, and detected by using an ECL blotting analysis system (Amersham Pharmacia Biotech, Piscataway, NJ).

\section{Tissue samples and immunohistochemistry}

Fresh lung tumor tissues were obtained from patients who were undergoing surgical resection of a primary tumor. All human tissue samples were obtained and analyzed in accordance with procedures approved by the institutional review board of the University of California, San Francisco (IRB H8714-22 942-01). The tissue microarray sections were immunostained as previously described [48]. The following scoring system was used: - , no stain; + , weak staining $(10 \%$ or above stained cellularity considered as positive); ++, moderate staining $(30 \%$ or above stained cellularity considered as positive); +++, strong staining (50\% or above stained cellularity considered as positive). All scoring was done under low power objective lens $(10 \times)$ with a Zeiss Axioscop 2 microscope (Carl Zeiss Inc, Germany). Images were taken under $10 \times$ or $20 \times$ objective lens.

\section{Immunofluorescence}

Cells were seeded on a Chamber SlideTM (Thermo Fisher Scientific) at 50\% confluence and fixed with $100 \%$ methanol following their respective treatments. After permeabilization using Triton-X100, slides were subsequently blocked for $1 \mathrm{~h}$ at room temperature with Dulbecco's phosphate-buffered saline (PBS) containing $5 \%$ bovine serum albumin (BSA) and incubated with primary antibody overnight at $4^{\circ} \mathrm{C}$. Antibodies were diluted in PBS containing 5\% BSA. Primary antibodies of YAP and phospho-YAP (Ser127) were purchased from Cell Signaling, Inc. (Danvers, MA). After being washed, the slides were incubated with the corresponding secondary antibodies in the dark for $1 \mathrm{~h}$ at room temperature, washed again, and mounted using ProLong Antifade with DAPI to visualize the nuclei. The slides were analyzed using a fluorescent confocal microscope equipped with an ultraviolet laser (Carl Zeiss Inc, Germany).

\section{ChIP assay}

Fragmented chromatin from SKLU-1 and H1299 cells was incubated with anti-IgG (negative control), anti-YAP, and anti-POL-II (positive control). Fragmented chromatin from SKLU-1, H1299, H1975 and A549 cells was incubated with anti-YAP. Recruited DNA was subjected to PCR using the primers for distal enhancer regions of PD-L1, and PCR products were electrophoresed in agarose gel. The ChIP assay was conducted using the Chromatin Immunoprecipitation (ChIP) Assay Kit (Millipore Corporation). Monoclonal antibodies for YAP (Cell Signaling Technology) and control rabbit antibody for IgG (Cell Signaling Technology) were used for ChIP. Two pairs' primer used for RT-PCR to amplify the PD-L1 gene, one pair primers were 5'-TCGGTCTGTGAAGGACT GC-3' and 5'-ACCGTTGAGGAATGGATGAA-3' resulting in a product size of $203 \mathrm{bp}$, and the other were 5'-CCACCACCATTATCTAATTCCA-3' and 5'-AAGGAG CCAGACACAAAAGG-3' resulting in a product size of $210 \mathrm{bp}$.

\section{Statistical analysis}

Data are expressed as mean \pm standard deviation (SD) from independent experiments. All statistical analyses were performed using the SPSS 23.0 for Windows software system (SPSS Inc, Chicago, IL). One-way ANOVA followed by Scheffe multiple comparisons were used to compare the differences among multiple groups. Statistical significance of differences between groups was determined by Student's $t$-test. The relationship between YAP and PD-L1 was analyzed with Spearman product correlation. For all analyses, statistical significance was defined as $P<0.05\left({ }^{*} P<0.05,{ }^{* *} P \leq 0.01\right.$, $\left.{ }^{* * *} P \leq 0.001\right)$, based on two-tailed tests.

\section{Author contributions}

JBM, PCH, DJ and LY conceived and designed the experiments. JBM and PCH carried out experiments. JBM analyzed the data. YLY, YYD, ZH, GC, BH, YCW and LH contributed materials tools. JBM organized and wrote the manuscript. LY, ZDX and YLY, reviewed and revised the manuscript. DJ gave important directions to the study and revised the manuscript. All authors had final approval of the submitted and published versions.

\section{ACKNOWLEDGMENTS}

We thank Pamela Derish in the UCSF Department of Surgery for editorial assistance with the manuscript.

\section{CONFLICTS OF INTEREST}

The authors declare no competing financial interests.

\section{FUNDING}

This study was supported by the National Institutes of Health (NIH; Grant No. R01 CA140654, to LY). We are grateful for support from the Kazan McClain 
Partners' Foundation; the Estate of Robert Griffiths; the Jeffrey and Karen Peterson Family Foundation; Paul and Michelle Zygielbaum; the Estate of Norman Mancini; and the Barbara Isackson Lung Cancer Research Fund. And the Basic and Clinical Cooperation Research Fund of China Capital Medical University (15JL39) and the Clinical Scientific Research Fund of Wu Jieping Medical Foundation $(320,6750,16217)$.

\section{REFERENCES}

1. Siegel R, Naishadham D, Jemal A. Cancer statistics, 2013. CA Cancer J Clin. 2013; 63:11-30.

2. Chen W, Zheng R, Baade PD, Zhang S, Zeng H, Bray F, Jemal A, Yu XQ, He J. Cancer statistics in China, 2015. CA Cancer J Clin. 2016; 66:115-132.

3. Ettinger DS, Wood DE, Aisner DL, Akerley W, Bauman J, Chirieac LR, D'Amico TA, DeCamp MM, Dilling TJ, Dobelbower M, Doebele RC, Govindan R, Gubens MA, et al. Non-Small Cell Lung Cancer, Version 5.2017, NCCN Clinical Practice Guidelines in Oncology. J Natl Compr Canc Netw. 2017; 15:504-535.

4. Lazzari C, Bulotta A, Ducceschi M, Viganò MG, Brioschi E, Corti F, Gianni L, Gregorc V. Historical Evolution of Second-Line Therapy in Non-Small Cell Lung Cancer. Front Med (Lausanne). 2017; 4:4.

5. Masters GA, Temin S, Azzoli CG, Giaccone G, Baker S Jr, Brahmer JR, Ellis PM, Gajra A, Rackear N, Schiller JH, Smith TJ, Strawn JR, Trent D, Johnson DH, and American Society of Clinical Oncology Clinical Practice. Systemic therapy for stage IV non-small-cell lung cancer: american society of clinical oncology clinical practice guideline update. J Clin Oncol. 2015; 33:3488-515.

6. Keir ME, Butte MJ, Freeman GJ, Sharpe AH. PD-1 and its ligands in tolerance and immunity. Annu Rev Immunol. 2008; 26:677-704.

7. Cortez MA, Ivan C, Valdecanas D, Wang X, Peltier HJ, Ye Y, Araujo L, Carbone DP, Shilo K, Giri DK, Kelnar K, Martin D, Komaki R, et al. PDL1 Regulation by p53 via miR-34. J Natl Cancer Inst. 2015; 108:djv303.

8. Yang H, Chen H, Luo S, Li L, Zhou S, Shen R, Lin H, Xie X. The correlation between programmed deathligand 1 expression and driver gene mutations in NSCLC. Oncotarget. 2017; 8:23517-28. https://doi.org/10.18632/ oncotarget. 15627.

9. Lee SJ, Jang BC, Lee SW, Yang YI, Suh SI, Park YM, Oh S, Shin JG, Yao S, Chen L, Choi IH. Interferon regulatory factor-1 is prerequisite to the constitutive expression and IFN-gamma-induced upregulation of B7-H1 (CD274). FEBS Lett. 2006; 580:755-762.

10. Couzin-Frankel J. Breakthrough of the year 2013. Cancer immunotherapy. Science. 2013; 342:1432-1433.

11. Mellman I, Coukos G, Dranoff G. Cancer immunotherapy comes of age. Nature. 2011; 480:480-489.
12. Zou W, Chen L. Inhibitory B7-family molecules in the tumour microenvironment. Nat Rev Immunol. 2008; 8:467-477.

13. Cooper WA, Tran T, Vilain RE, Madore J, Selinger CI, Kohonen-Corish M, Yip P, Yu B, O'Toole SA, McCaughan BC, Yearley JH, Horvath LG, Kao S, et al. PD-L1 expression is a favorable prognostic factor in early stage nonsmall cell carcinoma. Lung Cancer. 2015; 89:181-188.

14. Moroishi T, Hansen CG, Guan KL. The emerging roles of YAP and TAZ in cancer. Nat Rev Cancer. 2015; 15:73-79.

15. Dai Y, Liu S, Zhang WQ, Yang YL, Hang P, Wang H, Cheng L, Hsu PC, Wang YC, Xu Z, Jablons DM, You L. YAP1 regulates $\mathrm{ABCG} 2$ and cancer cell side population in human lung cancer cells. Oncotarget. 2017; 8:4096-109. https://doi.org/10.18632/oncotarget.13686.

16. Ma K, Xu Q, Wang S, Zhang W, Liu M, Liang S, Zhu H, $\mathrm{Xu}$ N. Nuclear accumulation of Yes-Associated Protein(YAP) maintains the survival of doxorubicininduced senescent cells by promoting survivin expression. Cancer Lett. 2016; 375:84-91.

17. Xiao L, Shi XY, Zhang Y, Zhu Y, Zhu L, Tian W, Zhu BK, Wei ZL. YAP induces cisplatin resistance through activation of autophagy in human ovarian carcinoma cells. Onco Targets Ther. 2016; 9:1105-1114.

18. Garcia-Rendueles ME, Ricarte-Filho JC, Untch BR, Landa I, Knauf JA, Voza F, Smith VE, Ganly I, Taylor BS, Persaud Y, Oler G, Fang Y, Jhanwar SC, et al. NF2 Loss Promotes Oncogenic RAS-Induced Thyroid Cancers via YAP-Dependent Transactivation of RAS Proteins and Sensitizes Them to MEK Inhibition. Cancer Discov. 2015; 5:1178-1193.

19. Gebhardt T, Harvey KF. Hippo Wades into Cancer Immunology. Dev Cell. 2016; 39:635-637.

20. Wang S, Xie F, Chu F, Zhang Z, Yang B, Dai T, Gao L, Wang L, Ling L, Jia J, van Dam H, Jin J, Zhang L, Zhou F. YAP antagonizes innate antiviral immunity and is targeted for lysosomal degradation through IKK $\varepsilon$-mediated phosphorylation. Nat Immunol. 2017; 18:733-43.

21. Zhao B, Ye X, Yu J, Li L, Li W, Li S, Yu J, Lin JD, Wang CY, Chinnaiyan AM, Lai ZC, Guan KL. TEAD mediates YAP-dependent gene induction and growth control. Genes Dev. 2008; 22:1962-71.

22. Mino-Kenudson M. Programmed cell death ligand-1 (PD-L1) expression by immunohistochemistry:could it be predictive and/or prognostic in non-small cell lung cancer? Cancer Biol Med. 2016; 13:157-170.

23. Steinhardt AA, Gayyed MF, Klein AP, Dong J, Maitra A, Pan D, Montgomery EA, Anders RA. Expression of Yesassociated protein in common solid tumors. Hum Pathol. 2008; 39:1582-1589.

24. Wang Y, Dong Q, Zhang Q, Li Z, Wang E, Qui X. Overexpression of yes-associated protein contributes to progression and poor prognosis of non-small-cell lung cancer. Cancer Sci.2010; 101:1279-1285. 
25. Sznol M, Chen L. Antagonist antibodies to PD-1 and B7-H1 (PD-L1) in the treatment of advanced human cancer. Clin Cancer Res. 2013; 19:1021-1034.

26. Mu CY, Huang JA, Chen Y, Chen C, Zhang XG. High expression of PD-L1 in lung cancer may contribute to poor prognosis and tumor cells immune escape through suppressing tumor infiltrating dendritic cells maturation. Med Oncol. 2011; 28:682-688.

27. Jiang L, Su X, Zhang T, Yin X, Zhang M, Fu H, Han H, Sun Y, Dong L, Qian J, Xu Y, Fu X, Gavine PR, et al. PD-L1 expression and its relationship with oncogenic drivers in non-small cell lung cancer (NSCLC). Oncotarget. 2017; 8:26845-57. https://doi.org/10.18632/oncotarget.15839.

28. Tschaharganeh DF, Chen X, Latzko P, Malz M, Gaida MM, Felix K, Ladu S, Singer S, Pinna F, Gretz N, Sticht C, Tomasi ML, Delogu S, et al. Yes-associated protein up-regulates Jagged-1 and activates the Notch pathway in human hepatocellular carcinoma. Gastroenterology. 2013;144:1530-1542.

29. Chen N, Fang W, Zhan J, Hong S, Tang Y, Kang S, Zhang Y, He X, Zhou T, Qin T, Huang Y, Yi X, Zhang L. Upregulation of PD-L1 by EGFR Activation Mediates the Immune Escape in EGFR-Driven NSCLC: Implication for Optional Immune Targeted Therapy for NSCLC Patients with EGFR Mutation. J Thorac Oncol. 2015; 10:910-923.

30. Dewey EB, Sanchez D, Johnston CA. Warts phosphorylates mud to promote pins-mediated mitotic spindle orientation in Drosophila, independent of Yorkie. Curr Biol. 2015; 25:2751-2762.

31. Johnson R, Halder G. The two faces of Hippo: targeting the Hippo pathway for regenerative medicine and cancer treatment. Nat Rev Drug Discov. 2014; 13:63-67.

32. Oudhoff MJ, Braam MJ, Freeman SA, Wong D, Rattray DG, Wang J, Antignano F, Snyder K, Refaeli I, Hughes MR, McNagny KM, Gold MR, Arrowsmith CH, et al. SETD7 Controls Intestinal Regeneration and Tumorigenesis by Regulating Wnt/ $\beta$-Catenin and Hippo/YAP Signaling. Dev Cell. 2016; 37:47-57.

33. Harvey KF, Zhang X, Thomas DM. The Hippo pathway and human cancer. Nat Rev Cancer. 2013; 13: 246-257.

34. Lamar JM, Stern P, Liu H, Schindler JW, Jiang ZG, Hynes RO. The Hippo pathway target, YAP, promotes metastasis through its TEAD-interaction domain. Proc Natl Acad Sci USA. 2012; 109:E2441-2450.

35. Bora-Singhal N, Nguyen J, Schaal C, Perumal D, Singh S, Coppola D, Chellappan S. YAP1 Regulates OCT4 Activity and SOX2 Expression to Facilitate Self-Renewal and Vascular Mimicry of Stem-Like Cells. Stem Cells. 2015; 33:1705-1718.

36. Overholtzer M, Zhang J, Smolen GA, Muir B, Li W, Sgroi DC, Deng CX, Brugge JS, Haber DA. Transforming properties of YAP, a candidate oncogene on the chromosome 11q22 amplicon. Proc Natl Acad Sci U S A. 2006; 103:12405-12410.
37. Zender L, Spector MS, Xue W, Flemming P, CordonCardo C, Silke J, Fan ST, Luk JM, Wigler M, Hannon GJ, $\mathrm{Mu} \mathrm{D}$, Lucito R, Powers $\mathrm{S}$, et al. Identification and validation of oncogenes in liver cancer using an integrative oncogenomic approach. Cell. 2006; 125:1253-1267.

38. Wang $\mathrm{X}, \mathrm{Su} \mathrm{L}, \mathrm{Ou} \mathrm{Q}$. Yes-associated protein promotes tumour development in luminal epithelial derived breast cancer. Eur J Cancer. 2012; 48:1227-1234.

39. Hsu PC, You B, Yang YL, Zhang WQ, Wang YC, Xu Z, Dai Y, Liu S, Yang CT, Li H, Hu B, Jablons DM, You L. YAP promotes erlotinib resistance in human non-small cell lung cancer cells. Oncotarget. 2016; 7:51922-33. https:// doi.org/10.18632/oncotarget.10458.

40. Lee BS, Park DI, Lee DH, Lee JE, Yeo MK, Park YH, Lim DS, Choi W, Lee DH, Yoo G, Kim HB, Kang D, Moon JY, et al. Hippo effector YAP directly regulates the expression of PD-L1 transcripts in EGFR-TKI-resistant lung adenocarcinoma. Biochem Biophys Res Commun. 2017; 491:493-99.

41. Pardoll DM. The blockade of immune checkpoints in cancer immunotherapy. Nat Rev Cancer. 2012; 12:252-264.

42. Dong H, Strome SE, Salomao DR, Tamura H, Hirano F, Flies DB, Roche PC, Lu J, Zhu G, Tamada K, Lennon VA, Celis E, Chen L. Tumor-associated B7-H1 promotes T-cell apoptosis: a potential mechanism of immune evasion. Nat Med. 2002; 8:793-800.

43. Loke P, Allison JP. PD-L1 and PD-L2 are differentially regulated by Th1 and Th2 cells. Proc Natl Acad Sci USA. 2003; 100:5336-5341.

44. Ritprajak P, Azuma M. Intrinsic and extrinsic control of expression of the immunoregulatory molecule PD-L1 in epithelial cells and squamous cell carcinoma. Oral Oncol. 2015; 51:221-228.

45. Feng J, Yang H, Zhang Y, Wei H, Zhu Z, Zhu B, Yang M, Cao W, Wang L, Wu Z. Tumor cell-derived lactate induces TAZ-dependent upregulation of PD-L1 through GPR81 in human lung cancer cells. Oncogene. 2017; 36:5829-39. https://doi.org/10.1038/onc.2017.188.

46. Bae JS, Kim SM, Lee H. The Hippo signaling pathway provides novel anti-cancer drug targets. Oncotarget. 2017; 8:16084-98. https://doi.org/10.18632/oncotarget.14306.

47. Chaib I, Karachaliou N, Pilotto S, Codony Servat J, Cai X, Li X, Drozdowskyj A, Servat CC, Yang J, Hu C, Cardona AF, Vivanco GL, Vergnenegre A, et al. Co-activation of STAT3 and YES-Associated Protein 1 (YAP1) Pathway in EGFR-Mutant NSCLC. J Natl Cancer Inst. 2017; 109. https://doi.org/10.1093/jnci/djx014.

48. Li T, Hou SC, Mao JH, Wang YC, Lu XD, Tan JL, You B, Liu YP, Ni J, Au A, Jablons DM, Xu Z, You L. The expression of Dishevelled-3 and glutamine metabolism in malignant pleural mesothelioma. J Clin Pathol. 2012; 65:855-858. 